\title{
Designing vaccines to prevent breast cancer recurrence or invasive disease
}

\author{
"Although breast cancer has not historically been believed to be an \\ immunogenic tumor, the importance of the role of the tumor immune \\ environment is emerging for both in invasive and preinvasive breast \\ cancer."
}

Keywords: antigen $\bullet$ breast cancer prevention $\bullet$ MHC Class II $\bullet$ Th1 • vaccine

\section{Introduction}

With the emerging importance of the tumor immune environment in both invasive and preinvasive breast cancer, vaccine therapy may provide a well-tolerated, durable therapy for preinvasive breast cancer by preventing both recurrent preinvasive disease and progression to invasive disease. Preinvasive breast cancer is ideal for vaccines because the disease is slow growing and the patients are not immunosuppressed. However, design of the vaccines are critical including consideration of appropriate antigens, selecting Th1 stimulating epitopes, and using multiantigen vaccines to address tumor heterogeneity.

\section{The importance of the immune} system in preinvasive breast cancer

Although breast cancer has not historically been believed to be an immunogenic tumor, the importance of the role of the tumor immune environment is emerging for both in invasive and preinvasive breast cancer. In invasive breast cancer (IBC), increased cytotoxic $\mathrm{CD}^{+}{ }^{+}$T-cell infiltrate shows improved breast cancer specific survival [1] whereas increased $\mathrm{FOXP3}^{+}$T-cell immune suppressive infiltrate is associated with inferior survival [2]. On examining specific breast cancer subtypes, a subset of triple negative breast cancer defined by robust immune infiltrate shows similar prognosis to luminal A tumors [3]. Preinvasive tumors and high-risk breast lesions also have increased immune infiltrate seen as early as benign proliferative breast disease, for example, $\mathrm{CD}^{+} \mathrm{T}$-cell infiltration increases from the mean in normal breast of $2.8 \pm 1.0$ to $81.5 \pm 14.0$ in benign proliferative breast disease, to $84.0 \pm 14.9$ in ductal carcinoma in situ (DCIS) and to $103.7 \pm 3.9$ in IBC [4]. Similar to invasive breast cancer, the populations of immune cells present in preinvasive disease are important for prognosis. A study of 62 DCIS tumors showed that increased $\mathrm{FOXP}^{+}{ }^{+}$infiltrate conferred a shorter relapse-free survival (HR: 2.81; 95\% CI: 0.99-7.99; $p=0.05$ ) [5]. Furthermore, gene expression profiling studies demonstrate that a chronic inflammatory signature is present in both DCIS and IBC [6], and a T helper 1 (Th1) proinflammatory signature demonstrated improved outcome as compared with a T helper 2 (Th2) immune suppressive signature $(\mathrm{p}=0.0394)$ [7]. These data suggest that as early as preinvasive disease, the immune system mounts an adaptive immune response to the tumor; therefore, if the immune system can be stimulated to mount the appropriate antitumor Th1 immune response to these preinvasive tumors it may be possible to prevent recurrence of the preinvasive lesions or progres-

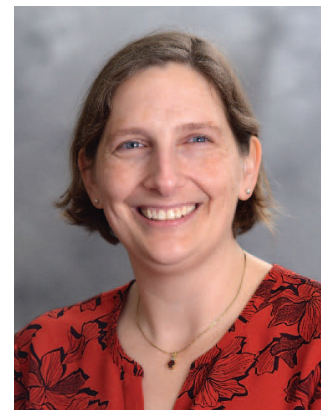

Sasha E Stanton

Author for correspondence:

Tumor Vaccine Group, Center for Translational Medicine in Women's Health, 850 Republican Street, 2nd Floor, Box 358050, University of Washington, Seattle, WA 98195-8050, USA

Tel.: +1 2066858849

Fax: +12066853128

sestant2@uw.edu

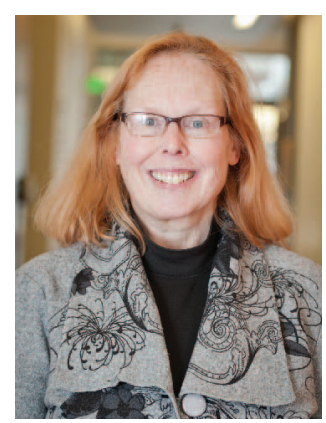

Mary L Disis

Tumor Vaccine Group, Center for Translational Medicine in Women's Health, 850 Republican Street, 2nd Floor, Box 358050, University of Washington, Seattle, WA 98195-8050, USA

Future : $\because$ Medicine part of 
sion to invasive cancer. Vaccine therapy should work well for patients with preinvasive breast cancer because these patients have slow growing disease, a low tumor burden and do not have a suppressed immune system. However for vaccine therapy to be effective, the vaccine has to be carefully designed to identify antigens that are present in preinvasive disease, the vaccine has to be designed to trigger a Th1 immune response and the heterogeneity of preinvasive breast cancer has to be addressed by targeting multiple immunogenic proteins present in the lesion.

\section{Identifying appropriate preinvasive vaccine antigens}

Antigen targets for preinvasive breast cancer should be immunogenic proteins that are overexpressed in highrisk DCIS or other preinvasive breast lesions and are associated with an increased risk of developing invasive breast cancer [8]. Overexpressed proteins are believed to be immunogenic by revealing cryptic epitopes and by increasing the number of peptides available for recognition by the immune system [9]. Three examples of possible preinvasive antigen targets include IGF1R, IGFBP2 and HER2. IGF1R is overexpressed in both preinvasive and invasive breast cancer and has increased autoantibodies in breast cancer patients as compared with normal donors $(\mathrm{p}<0.04)$ [10]. Increased autoantibodies to IGFBP2 have been seen in breast cancer patients over controls ( $\mathrm{p}=0.0008)$ and IGFBP2 has been shown to be important in pathogenesis of both preinvasive and invasive breast cancer [11]. IgG HER2 specific antibodies are found to be increased in patients with the HER2 ${ }^{+}$breast cancer subtype, and patients with high levels of IFN- $\gamma$ secreting HER2specific $\mathrm{T}$ cells are significantly more likely to have $3+$ overexpressing tumors $(\mathrm{p}=0.001)$ [12]. Furthermore, HER2 is also found overexpressed in DCIS and confers a higher risk of progressing to invasive disease [13]. Importantly, all three of these preinvasive antigens are also overexpressed in invasive breast cancer; therefore, a vaccine against these targets should not only prevent recurrence of preinvasive disease but also prevent against developing invasive disease.

\section{Designing vaccines to produce a Th1 immune response}

The proinflammatory Th1 immune environment promotes the best antitumor response; however, most tumors stimulate a Th2 immunosuppressive environment [14]. For example, PBMC from breast cancer patients had an increased Th 2 response to MHC class II predicted IGF1R epitopes as compared to matched normal controls [10]. Th1 immune response both destroys the current disease and provides immunologic memory to protect against recurrence or progression because of IFN- $\gamma$ associated inflammatory cytokines which are necessary for enhanced function of antigen presenting cells, epitope spreading, activation of the $\mathrm{CD} 8^{+} \mathrm{T}$ cells and immunologic memory [15,16]. Using MHC class II vaccines which stimulate Th1 $\mathrm{CD} 4^{+} \mathrm{T}$ cells can both increase recruitment of $\mathrm{CD} 8^{+} \mathrm{T}$ cells to the tumor and allow them to be active by promoting a Th1 immune environment [17]. In the preclinical transgenic mouse mammary tumor model TgMMTV-neu, an MHC class II vaccine against IGFBP2 could inhibit tumor growth and this antitumor function was $\mathrm{CD}^{+}{ }^{+} \mathrm{T}$-cell dependent [11]. Furthermore, MHC class II epitopes from the same protein can either stimulate a predominantly IFN- $\gamma$ Th1 or IL-10 Th2 response and inclusion of any Th 2 epitopes in a vaccine prevents the antitumor immune response. For example, in the protein IGFBP2, the $\mathrm{N}$ terminus stimulated predominantly a Th1 IFN- $\gamma$ immune response and as a vaccine could inhibit tumor growth in the transgenic TgMMTVneu mice. However, when the vaccine included both the $\mathrm{N}$ terminus and the $\mathrm{C}$ terminus (which stimulated predominantly a Th2 response) there was no tumor inhibition [18]. For effective prevention vaccines, epitopes need to be selected that primarily promote a Th1 response to avoid the immunosuppressive Th2 epitopes that prevent an antitumor immune response.

“

"...studies give preclinical evidence that a multiantigen vaccine using Th1-specific MHC class II epitopes can be effective at preventing development of both preinvasive and invasive breast cancer. The immunogenicity of this vaccine in women will soon be tested in a clinical trial."

\section{Multiantigen vaccines to address tumor heterogeneity}

Preinvasive breast tumors are heterogeneous, therefore, there is differential expression of target proteins even in the same premalignant lesion [19]. Since HER2 had been shown to be both overexpressed and immunogenic in preinvasive breast cancer, a dendritic cell vaccine directed against HER2 was tested in patients with HER2+ DCIS [13]. The HER2 vaccine was immunogenic and $91 \%$ of the subjects had greater than fivefold response to HER 2 peptides by IFN- $\gamma$ ELISPOT after vaccination. Furthermore, there were increased HER2 peptide responsive $\mathrm{CD}^{+} \mathrm{T}$ cells in DCIS tissue by FACS in $88 \%$ of patients after vaccination. However, $84.6 \%$ of the patients had residual DCIS, although $55 \%$ of patients had decrease in size of DCIS, and the DCIS remaining had lost HER2 expression [13]. Using a single antigen vaccine allowed for selection of clones that do not overexpress the particular immunologic 
target. Furthermore, HER 2 is only expressed in a subset $(\sim 20 \%)$ of DCIS; therefore, single antigen HER2 vaccines only address a subset of DCIS.

A preclinical multivalent vaccine developed by our group has demonstrated that vaccination with a vaccine containing HER2, IGF-IR, and IGFBP2 epitopes can prevent both DCIS and progression from DCIS to invasive breast cancer in the TgMMTV-neu transgenic mouse mammary tumor model which develop tumors similar to luminal B breast cancer [20]. When mice were immunized at approximately 18 weeks old, the multiantigen vaccine was able to block development of palpable lesions in $65 \%$ of the mice as compared with adjuvant alone $(\mathrm{p}<0.0001)$ and improve overall survival $(\mathrm{p}<0.0002)$ in TgMMTV-neu mice. Furthermore, while animals vaccinated with the single antigens had similar diseasefree survival $(p=0.965)$, the disease-free survival of the animals vaccinated with the multiantigen vaccine was significantly improved over any of the individual antigens $(p=0.018)$. The tumors that did develop in the multiantigen-vaccinated mice were slower growing and demonstrated increased $\mathrm{CD} 8^{+} \mathrm{T}$-cell tumor infiltration as compared with control-vaccinated mice $(p=0.003)$. Similar prevention of invasive disease was found when vaccinating the very aggressive C3(1) Tag transgenic mice which develop tumors that are genetically similar to triple negative breast cancer $(\mathrm{p}<0.0001)$. The protection was $\mathrm{CD}^{+}$mediated and depletion of the $\mathrm{CD}^{+} \mathrm{T}$ cells caused loss of the tumor inhibitory effect $(p=0.013)$ [20]. These studies give preclinical evidence that a multiantigen vaccine using Th1-specific MHC class II epitopes

\section{References}

1 Mahmoud SM, Paish EC, Powe DG et al. Tumor-infiltrating $\mathrm{CD} 8^{+}$lymphocytes predict clinical outcome in breast cancer. J. Clin. Oncol. 29(15), 1949-1955 (2011).

2 Ladoire S, Arnould L, Apetoh L et al. Pathologic complete response to neoadjuvant chemotherapy of breast carcinoma is associated with the disappearance of tumor-infiltrating foxp $3^{+}$ regulatory T cells. Clin. Cancer Res. 14(8), 2413-2420 (2008).

3 Loi S, Sirtaine N, Piette F et al. Prognostic and predictive value of tumor-infiltrating lymphocytes in a Phase III randomized adjuvant breast cancer trial in node-positive breast cancer comparing the addition of docetaxel to doxorubicin with doxorubicin-based chemotherapy: BIG 02-98. J. Clin. Oncol 31(7), 860-867 (2013).

4 Hussein MR, Elsers DA, Fadel SA, Omar AE. Immunohistological characterisation of tumour infiltrating lymphocytes in melanocytic skin lesions. J. Clin. Pathol. 59(3), 316-324 (2006).

5 Bates GJ, Fox SB, Han C et al. Quantification of regulatory $\mathrm{T}$ cells enables the identification of high-risk breast can be effective at preventing development of both preinvasive and invasive breast cancer. The immunogenicity of this vaccine in women will soon be tested in a clinical trial.

\section{Conclusion}

The emerging role of the immune system in breast cancer has shown that the quantity and composition of the immune response to the tumor affect prognosis even in preinvasive lesions. Using intelligent vaccine design to ensure that the vaccines can stimulate a Th1 immune environment and target multiple antigens has been shown to both prevent preinvasive and invasive disease in preclinical transgenic mouse models where without treatment all mice develop mammary tumors. Preinvasive breast cancer would be an ideal disease for vaccine therapy and vaccines would address an unmet need in these patients for a durable, safe, well-tolerated therapy that has the potential to prevent both recurrence and progression.

\section{Financial \& competing interests disclosure}

SE Stanton was supported by KL2TR000421. ML Disis was supported by the Athena Distinguished Professor of Breast Cancer Research, U01CA141539 and DOD W81XWH-11-1-0760. ML Disis holds a Komen Leadership Award and has several patents held by the University of Washington. The authors have no other relevant affiliations or financial involvement with any organization or entity with a financial interest in or financial conflict with the subject matter or materials discussed in the manuscript apart from those disclosed.

No writing assistance was utilized in the production of this manuscript.

cancer patients and those at risk of late relapse. J. Clin. Oncol 24(34), 5373-5380 (2006).

6 Muggerud AA, Hallett M, Johnsen $\mathrm{H}$ et al. Molecular diversity in ductal carcinoma in situ (DCIS) and early invasive breast cancer. Mol. Oncol. 4(4), 357-368 (2010).

7 Kristensen VN, Vaske CJ, Ursini-Siegel J et al. Integrated molecular profiles of invasive breast tumors and ductal carcinoma in situ (DCIS) reveal differential vascular and interleukin signaling. Proc. Natl Acad. Sci. USA 109(8), 2802-2807 (2012).

8 Kerlikowske K, Molinaro AM, Gauthier ML et al. Biomarker expression and risk of subsequent tumors after initial ductal carcinoma in situ diagnosis. J. Natl Cancer Inst. 102(9), 627-637 (2010).

9 Nanda NK, Sercarz EE. The positively selected T cell repertoire: is it exclusively restricted to the selecting MHC? Int. Immunol. 7(3), 353-358 (1995).

10 Cecil DL, Park KH, Gad E et al. T-helper I immunity, specific for the breast cancer antigen insulin-like growth 
factor-I receptor (IGF-IR), is associated with increased adiposity. Breast Cancer Res. Treat. 139(3), 657-665 (2013).

11 Park KH, Gad E, Goodell V et al. Insulin-like growth factorbinding protein-2 is a target for the immunomodulation of breast cancer. Cancer Res. 68(20), 8400-8409 (2008).

12 Goodell V, Waisman J, Salazar LG et al. Level of HER-2/ neu protein expression in breast cancer may affect the development of endogenous HER-2/neu-specific immunity. Mol. Cancer Ther. 7(3), 449-454 (2008).

13 Czerniecki BJ, Koski GK, Koldovsky U et al. Targeting HER$2 /$ neu in early breast cancer development using dendritic cells with staged interleukin-12 burst secretion. Cancer Res. 67(4), 1842-1852 (2007).

14 Denardo DG, Andreu P, Coussens LM. Interactions between lymphocytes and myeloid cells regulate pro- versus anti-tumor immunity. Cancer Metastasis Rev. 29(2), 309-316 (2010).

15 Vanderlugt CL, Miller SD. Epitope spreading in immunemediated diseases: implications for immunotherapy. Nat Rev. Immunol. 2(2), 85-95 (2002).
16 Castellino F, Germain RN. Cooperation between CD4 ${ }^{+}$ and $\mathrm{CD} 8{ }^{+} \mathrm{T}$ cells: when, where, and how. Ann. Rev. Immunol. 24, 519-540 (2006).

17 Cohen PA, Peng L, Plautz GE, Kim JA, Weng DE, Shu S. $\mathrm{CD}^{+} \mathrm{T}$ cells in adoptive immunotherapy and the indirect mechanism of tumor rejection. Crit. Rev. Immunol. 20(1), $17-56$ (2000).

18 Cecil DL, Holt GE, Park KH et al. Elimination of IL10-inducing T-helper epitopes from an IGFBP-2 vaccine ensures potent antitumor activity. Cancer Res. 74(10), 2710-2718 (2014).

19 Mokbel K, Cutuli B. Heterogeneity of ductal carcinoma in situ and its effects on management. Lancet. Oncol. 7(9), 756-765 (2006)

20 Disis ML, Gad E, Herendeen DR et al. A multiantigen vaccine targeting neu, IGFBP-2, and IGF-IR prevents tumor progression in mice with preinvasive breast disease. Cancer Prev. Res. 6(12), 1273-1282 (2013). 